\title{
Nightstick Fractures, Outcomes of Operative and Non-Operative Treatment
}

\author{
Mohammed Ali*, D. I. Clark, Amole Tambe
}

\begin{abstract}
Introduction: A nightstick fracture is an isolated fracture of the ulnar shaft. Although operative and non-operative treatments have been commonly decided by the degree of displacement of the fracture, still there is a controversy specially in those moderately displaced. Herein we report our experience with nightstick fractures.

Objective: To evaluate operative and non-operative treatment of nightstick fracture.

Materials and methods: We retrospectively reviewed the clinical notes, physiotherapy letters and radiographs of 52 patients with isolated ulnar shaft fractures. Outcome Measurements included radiographic healing, post-operative range of motion and complications. Results: The study included 13 females and 39 males, with a mean age of 26 years [range, 18-93 years]. The mean Follow-up period was 32 months ranged from 12 to 54 months. Ten patients were treated non-operatively; forty-two patients had open reduction and internal fixation including six open fractures. The average wait for surgery was 2.5 days. Mobilisation was commenced immediately after the surgeries non-load bearing. 40 patients had no complications post-operatively with good outcome and average of four visits follow-up. In the non-operative group, five out ten failed and had a mean follow-up of nine visits.

Conclusion: Satisfactory outcome is to be expected with open reduction and internal fixation. Fractures with less than $50 \%$ displacement should be treated on individual bases, considering; age, pre-morbid functional status, co-morbidities, compliance and associated injuries.
\end{abstract}

\section{KEYWORDS}

nightstick fracture; ulnar shaft fracture; non-operative management; non-union

\section{AUTHOR AFFILIATIONS}

Royal Derby Hospital, Derby, United Kingdom

* Corresponding author: Trauma and Orthopaedics Department, South Tyneside District Hospital, Harton Ln, South Shields NE34 OPL, United Kingdom; e-mail: mohammedkhider84@hotmail.com

Received: 14 January 2018

Accepted: 8 November 2018

Published online: 1 April 2019

Acta Medica (Hradec Králové) 2019; 62(1): 19-23

https://doi.org/10.14712/18059694.2019.41

(c) 2019 The Authors. This is an open-access article distributed under the terms of the Creative Commons Attribution License (http://creativecommons.org/licenses/by/4.0), which permits unrestricted use, distribution, and reproduction in any medium, provided the original author and source are credited. 


\section{INTRODUCTION}

A nightstick fracture is an isolated fracture of the ulnar shaft (IUSF) associated with a direct blow usually as a result of the forearm being held in protection across the face (1). It can also occur with excessive supination or pronation. In these fractures, the integrity of the periosteum and the interosseous membrane determines the stability of the fracture and this is normally indicated by the initial displacement. Although typically closed fractures, they have a higher rate of delayed union or non-union. The aim of management is to prevent the complications of malunion and non-union and restore the best possible function of the limb. Majority of nightstick fractures used to be treated non-operatively (1) and numerous methods of immobilisation have been adopted by surgeons. However, the treatment of isolated ulnar fractures remains controversial, with different authors advocating both surgical and non- surgical management. Herein we report our experience with nightstick fractures.

\section{METHODS}

Retrospectively, we reviewed 96 consecutive patients with ulnar shaft fractures admitted to our hospital from September 2010 to December 2015. The study comprised review of patients' clinical notes and radiographs. Ulnar shaft fractures with ipsilateral radial, humeral, or wrist fractures were excluded. Add to that, Monteggia fractures were excluded, as were fractures of the olecranon or coronoid or styloid processes. These fractures resulted from assaults with a stick like weapon, road accidents or falls. 52 patients met the inclusion criteria. The method of treatment was decided by the treating surgeon. Part of these fractures was treated with open reduction and internal fixation using plates and the other part were treated closed with an above elbow arm cast.

We also reviewed the site of these fractures (proximal, middle, or distal shaft) and the degree of displacement.

Surgeries were performed either under general anaesthesia with local anaesthetic infiltration or regional block. Patients were placed in a supine position with the arm placed on an upholstered arm-board. Pneumatic tourniquet was used in all cases. An ulnar approach to the ulnar shaft was performed in all operative cases.

For antimicrobial prophylaxis, Cefuroxime was used intravenously, with $1.5 \mathrm{~g}$ injected intra-operatively and $0.75 \mathrm{~g}$ injected after 8 and 16 hours. The operated arm of all patients had a wool and crepe dressing and put in a broad arm sling post-operatively.

Patients started gentle wrist and shoulder movement non-load bearing immediately after surgery. Patients then seen after two weeks in outpatient clinics, where they had a wound check and started gentle elbow exercise including active pronation/ supination movement. All patients had post-operative physiotherapy referral initiated at two weeks as per our hospital protocol. Reduction and fixation were checked with plane radiographs at two weeks, six and 12 weeks post-operatively. Based on the progress with the physiotherapy and the bony union on the radiographs they either discharged with an open appointment or given further follow-up appointment.

In the non-operative group; immobilisation was obtained through an above elbow back-slab in a mid-prone position with the elbow in 90 degrees flexion and the wrist in a neutral position. After one week, patients had a repeat radiograph to check the position of the fracture. The backslab was then either completed or converted to a full cast at one week if the fracture position still acceptable. Patients had x-rays at two and four weeks in view of late slipping. Patients also had x-rays at 8 weeks and when there was good evidence of healing, cast was removed they were referred to physiotherapy. At 12 weeks, they will be reviewed again with plain radiographs to assess the healing of the fracture specially if patients are symptomatic.

Upon review, fracture union was considered when there is a bridging callus with no tenderness or movement at the fracture site. Non-union was declared when there was evidence of hypertrophic callus without bridging and persistent pain at the fracture site.

\section{RESULTS}

52 cases were found to be isolated ulnar shaft fractures. This included 13 females and 39 males, with a mean age of 26 years [range, $18-93$ years]. The mean Follow-up period was 32 months ranged from 12 months to 54 months. One patient had proximal third shaft fracture, 12 patients had distal shaft fractures and 39 patients demonstrated mid shaft fractures. 16 fractures were comminuted, 22 oblique displaced fractures, 13 transverse displaced fractures, one un-displaced transverse fracture. 6 patients had Open fractures.

\section{THE OPERATIVE GROUP}

42 patients had open reduction and internal fixation using plate and screw fixation, this group included six open fractures. 38 fractures were fixed using a dynamic compression plate (DCP) and four with a limited contact dynamic compression plate (LC-DCP) (Figure 1). The mean waiting time for surgery was 2.5 days, ranging from 1 to 7 days.

None developed wound infection or wound breakdown post-operatively. There were no recorded instances of nerve damage.

Adequate union was achieved in 40 (95\%) cases. Two patients (5\%) after DCP fixation, developed non-union during the follow-up (20 and 24 weeks) period and required a revision surgery (Figure 2). Fixation in both cases was done after anatomical reduction. Both patients had uneventful early post-operative course however one patient was reported to be a smoker and the other patient we could not find any mechanical or biological reason for the non-union.

In the 42 surgical cases; Anatomical reduction from time of surgery was maintained in all patients during follow-up. All patients were reported to have full supination, pronation and mean flexion arc of 10 to 130 degrees $(+/-10)$ at 12 Week. Good callus formation was noted in 40 patients at 12 weeks. No reported cases of mal-union or 


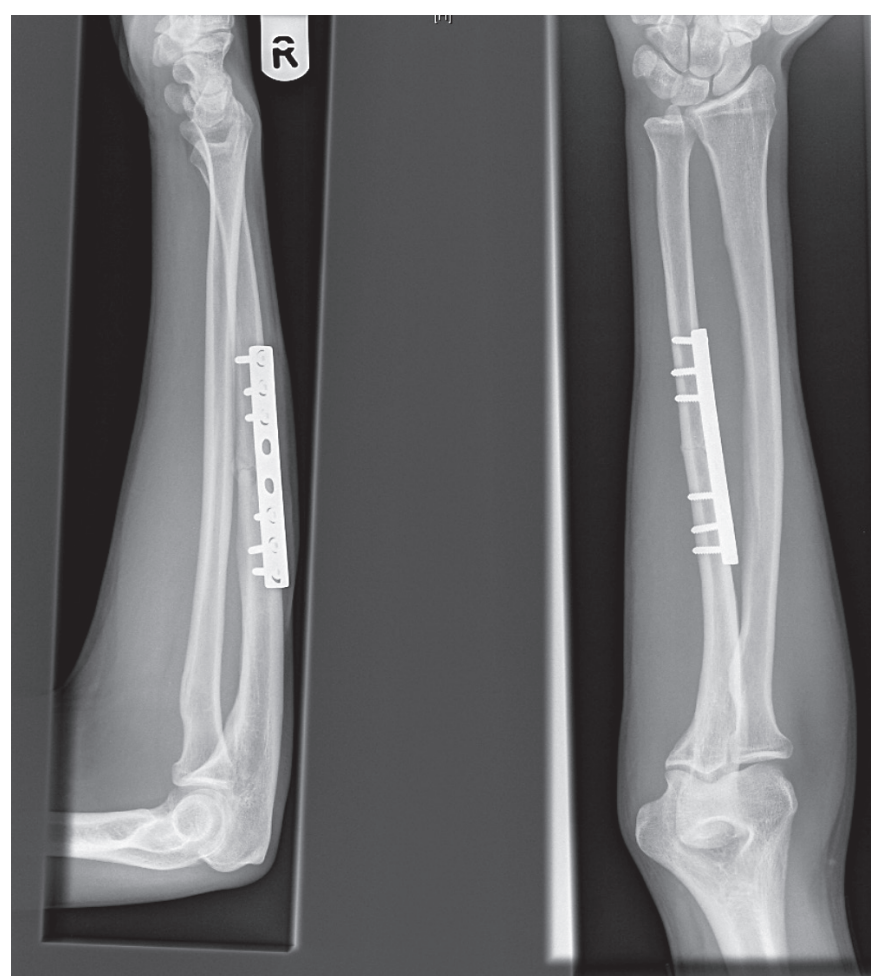

Fig. $1 \mathrm{x}$-rays show anatomical reduction of the fracture and fixation using plate and screws.

metal work failure. Only one patient was reported to develop metal work irritation from a DCP plate and required a metal work removal after 6 months. The average number of hospital visits was 4 (ranged from 4 to 6 visits).

\section{THE NON-OPERATIVE GROUP}

Ten patients were treated non-operatively (Table 1). Five patients were declared to fail non-operative treatment. One patient developed a mal-union however; he was happy with the function and did not want to go down the

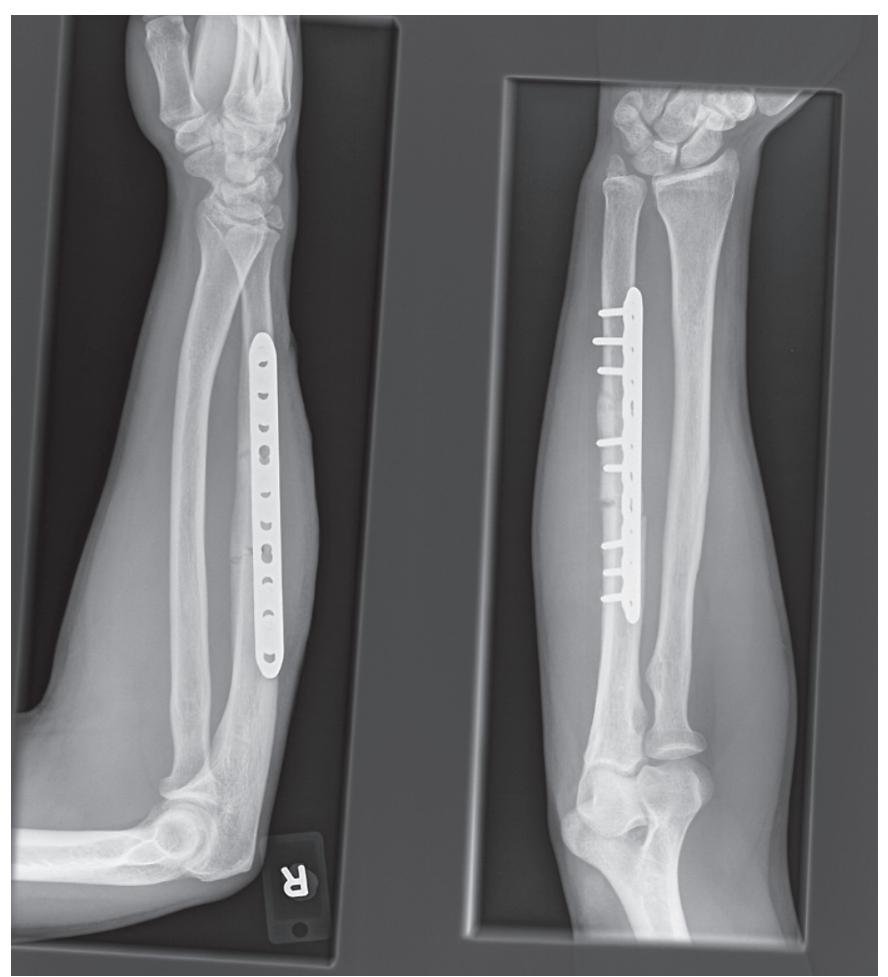

Fig. $2 \mathrm{X}$-rays show revision of a non-united fracture.

surgical route. At two weeks, four patients had further displacement beyond the acceptable limits. Three of them had a surgical fixation after two weeks and one patient was deemed not to be fit for surgery and went into non-union. The average number of hospital visits was 9 (ranged from 7 to 10 visits).

The other five patients had above elbow plaster cast in a mid-prone position. Cast was reduced to below elbow at 4 weeks and removed at eight weeks. Patients were then referred to physiotherapy. At 12 weeks, they were reviewed again with plain radiographs and fractures deemed to be

Tab. 1 Patients who received non-operative treatment.

\begin{tabular}{|l|l|l|l|l|l|l|}
\hline Age/sex & ASA & PMH & Smoking & Fracture & Treatment & Complications \\
\hline $73 / M$ & 4 & IHD, OP & No & Mid-shaft disp-oblique & Above elbow casting & Non-union \\
\hline $49 / M$ & 1 & None & No & Mid-shaft comminuted & Above elbow casting & None \\
\hline 89/M & 4 & OP, IHD, CKD & No & Mid-shaft comminuted & Above elbow casting & None \\
\hline $35 / F$ & 1 & None & No & Distal third disp-oblique & Above elbow casting & None \\
\hline $21 / M$ & 1 & None & Yes & Distal third disp-oblique & $\begin{array}{l}\text { Above elbow casting } \\
\text { / ORIF }\end{array}$ & $\begin{array}{l}\text { Further displacement } \\
\text { beyond acceptable limit }\end{array}$ \\
\hline $73 / M$ & 2 & PVD, OP & No & Mid-shaft disp-trans & Above elbow casting & None \\
\hline $59 / F$ & 3 & CKD, OP & No & Mid-shaft disp-trans & Above elbow casting & None \\
\hline $51 / F$ & 1 & None & No & Distal third disp-trans & $\begin{array}{l}\text { Above elbow casting } \\
\text { / ORIF }\end{array}$ & $\begin{array}{l}\text { Further displacement } \\
\text { beyond acceptable limit }\end{array}$ \\
\hline $32 / M$ & 1 & None & No & Mid-shaft disp-trans & $\begin{array}{l}\text { MUA / Above elbow } \\
\text { casting }\end{array}$ & Mal-union \\
\hline 18/M & 1 & None & No & Mid-shaft undisp-trans & $\begin{array}{l}\text { Above elbow casting } \\
\text { / ORIF }\end{array}$ & $\begin{array}{l}\text { Further displacement } \\
\text { beyond acceptable limit }\end{array}$ \\
\hline
\end{tabular}


united based on radiographs and clinical examination. $\mathrm{Pa}$ tients were reported to have ( -5 degrees) from full supination and pronation and mean flexion arc of 10 to 120 degrees (+/-10). At 12 weeks, all were discharged to the care of the physiotherapy and left with an open appointment should they have any problems in the future.

\section{DISCUSSION}

Non-operative treatment has been embraced by many authors and traditionally, benign neglect or non-operative management was reported to give satisfactory results with a prompt return to function and good healing rates. Traditionally, non-operative treatment was mainly recommended for non-displaced fractures or fractures with less than $50 \%$ displacement (2-4). The 1984 paper by Dymond et al. (5) which was authored based on a cadaveric study, concluded that more than $50 \%$ displacement involves considerable disruption of the periosteum and of the interosseous membrane. These displaced fractures were deemed to be unstable and necessitate above-elbow immobilisation for stability. In a minimally displaced ulnar shaft fracture, these structures, together with the intact radius, provide a strong stabilizing effect, which may explain why some authors $(6,7)$ were able to achieve satisfactory outcomes by treating low-energy ulnar fractures with minimum immobilization $(8,9)$. Furthermore, two studies have concluded that minimally displaced IUSFs in the middle and distal third of the ulna are stable and can be mobilised at earlier stages $(10,11)$. On the other hand, some authors suggested that proximal IUSF are best treated by ORIF, believing that the soft-tissue forces tend to destabilize fractures in this region. Also, it is possible that some of these are occult Monteggia fractures that have spontaneously reduced $(9,10)$. Hopper and Sarmiento although they advised non-operative treatment for diaphysial fractures in the distal two thirds, they excluded those in which the bone ends are displaced by $5 \mathrm{~mm}$ or more and advised to be treated with open reduction and internal fixation, particularly if the mechanism of injury was high energy $(2,11)$. Riska and Nottage, shared the same views however, they excluded patients with head injury, spinal cord injury, or poly-trauma patient in whom internal fixation of distal two-thirds ulnar fractures may facilitate acute care or rehabilitation $(12,13)$. Szabo et al. (14) retrospectively reviewed the treatment and outcome of 46 isolated fractures of the ulnar shaft. 18 fractures had internal fixation and 28 were treated closed. One open fracture became infected following fixation and failed to unite. Seven failed the non-operative treatment and ended up with non-union. They suggested prognostic factors for non-union in non-operatively treated fractures which inclde; proximal third fractures and those with displacement $5 \mathrm{~mm}$ or more.

Immobilisation positions have been discussed as having a great role in maintaining fracture reduction and the eventual acceptable alignment of the healed fracture. Traditionally, recommendations for immobilizing the forearm in neutral, supination, or pronation positions have been based on theory, anecdotal experience, and tradition (15-18). A few clinical studies have shown success with the forearm immobilized in either pronation or supination $(16,18)$. Altner et al. (19) in their series, they immobilised patients in a mid-prone position and reported good results. Add to that Boyer et al. (20) evaluated the effect of forearm position on the healing outcomes following non-surgical treatment using above elbow cast. They concluded that residual fracture angulation at the time of union was not significantly affected by forearm position.

A review by Mackay (21) et al. in 2000, included 33 series involving 1876 patients. The outcomes of the non-surgical treatment of minimally displaced ulnar fractures with a stable configuration were consistently satisfactory. Below elbow plaster cast, functional brace and early mobilisation all achieved similar results. Above elbow cast was deemed to be unnecessarily restrictive. Mackay also concluded that open reduction and internal fixation is better used in widely displaced or unstable fractures to preserve the forearm rotation.

Moed et al. (22) Reported the outcomes of immediate internal plate fixation of an open diaphyseal fracture of the forearm in fifty patients. Although they had two cases of deep infection and non-union in six, the functional results were excellent or good in 85 per cent of the series. They related these results to the severity of the initial soft-tissue injury and the surgical technique and recommended autogenous cancellous bone-grafting in comminuted fractures. On the other hand, Wright et al. (23) studied 198 forearm fractures to determine the union rate where acute bone grafting was recommended but not performed. The overall union rate in comminuted, non-grafted forearm fractures (open and closed) was $98 \%$. Another study by Wei et al. (24) concluded that acute bone grafting of diaphyseal forearm fractures did not affect the union rate or the time to union.

Recently, Cai and his fellow researchers (1) reviewed all published randomised controlled trials and observational studies that have assessed the outcome of these fractures following above- or below-elbow immobilisation, bracing and early mobilisation. They included 27 studies comprising 1629 fractures. They found that early mobilisation produced the shortest radiological union time and the lowest mean rate of non-union. They advised early mobilisation, with a removable forearm support for the treatment of nondisplaced or partially displaced nightstick fractures.

Coulibaly et al. in 2015 conducted a retrospective case-control analysis on patients diagnosed with IUSF to compare surgical and nonsurgical outcomes (25). They measured complications and functional ability. They found that nonsurgical treatment of IUSF is prone to complications and is associated with mal-union and non-union while Surgical treatment with rigid plate fixation and early range of motion resulted in a shorter period of cast immobilization and an earlier return to weight bearing, and led to reduced patient morbidity.

In our study, although the numbers are not comparable, complication rates in the non-operatively treated group were significantly higher than those reported in the operated group. In our study five fractures, Failed the non-operative treatment. Another notable correlate in 
our series was location in the middle third of the shaft, an experience not shared in previous papers. Also, patients who developed these complications either were very young and might not be very compliant or elderly with multiple co-morbidities and this was also noted by Coulibaly.

The limitations of our study include its retrospective nature, the small number of patients and the relatively short duration of follow-up. There are other elements that we did not measure and that could have contributed to our conclusion.

\section{CONCLUSION}

Based on our study and the published literature we believe that IUSFs with more than $5 \mathrm{~mm}$ displacement should be treated operatively. Satisfactory outcome is to be expected with open reduction and internal fixation with rigid plate as it allows early mobilisation and enables earlier return to function with very low risk of wound problems. Although our study did not reveal good results with non-operative treatment, we believe those with less than $5 \mathrm{~mm}$ displacement should be treated on individual bases, considering; age, pre-morbid functional status, co-morbidities, compliance and associated injuries.

\section{REFERENCES}

1. Cai XZ, Yan SG, Giddins G. A systematic review of the non-operative treatment of nightstick fractures of the ulna. Bone Joint J $2013 \mathrm{Jul}$; 95-B(7): 952-9.

2. Hooper G. Isolated fractures of the shaft of the ulna. Injury 1974; 6(2): 180-4.

3. Du Toit FP, Grabe RP. Isolated fractures of the shaft of the ulna. S Afr Med I 1979; 56(1): 21-5.

4. Corea JR, Brakenbury PH, Blakemore ME. The treatment of isolated fractures of the ulnar shaft in adults. Injury 1981; 12(5): 365-70.

5. Dymond IW. The treatment of isolated fractures of the distal ulna. J Bone Joint Surg (Br) 1984; 66(3): 408-10.

6. Pollock FH, Pankovich AM, Prieto JJ, Lorenz M. The isolated fracture of the ulnar shaft. Treatment without immobilization. J Bone Joint Surg Am 1983; 65(3): 339-42.

7. Sarmiento A. Isolated ulnar shaft fractures treated with functional braces. J Orthop Trauma 1998; $12(6): 420-3$.

8. Ekelund AL, Nilsson OS. Early mobilization of isolated ulnar shaft fractures. Acta Orthop Scand 1989; 60(3): 261-2.

9. de Jong T, de Jong PC. Ulnar shaft fracture needs no treatment. A pilot study of 10 cases. Acta Orthop Scand 1989; 60(3): 263-4.

10. Zych GA, Latta LL, Zagorski JB. Treatment of isolated ulnar shaft fractures with prefabricated functional fracture braces. Clin Orthop 1987; (219): 194-200.

11. Sarmiento A. Treatment of ulnar fractures by functional bracing. J Bone Joint Surg Am 1976; 58(8): 1104-7.

12. Nottage WM. A review of long bone fractures in patients with spinal cord injuries. Clin Orthop 1981; (155): 65-70.

13. Riska EB, von Bonsdorff H, Hakkinen S, Jaroma H, Kiviluoto O, Paavilainen T. Primary operative fixation of long bone fractures in patients with multiple injuries. J Trauma 1977; 17(2): 111-21.

14. Szabo RM, Skinner M. Isolated ulnar shaft fractures. Retrospective study of 46 cases. Acta Orthop Scand 1990 Aug; 61(4): 350-2.

15. Davis DR, Green DP. Forearm fractures in children. Clin Orthop 1976; 120: $172-84$.

16. Evans EM. Rotational deformity in the treatment of fractures of both bones of the forearm. J Bone Joint Surg 1945; 27: 373-9.

17. Gibbons CLMH, Woods DA, Pailthorpe C, et al. The management of isolated distal radius fractures in children. J Pediatr Orthop 1994; 14: 207-10.

18. Gupta RP, Danielsson LG. Dorsally angulated solitary metaphyseal greenstick fractures in the distal radius: Results after immobilization in pronated, neutral, and supinated position. J Pediatr Orthop 1990; 10: $90-2$

19. Altner, PC, Ted Hartman, J. Isolated Fractures of the Ulnar Shaft in the Adult. Surg Clin North Am 1972; 52 (1): 155-70.

20. Boyer BA, Overton B, Schrader W, Riley P, Fleissner P. Position of Immobilization for Pediatric Forearm Fractures. J Pediatr Orthop 2002; 22(2): 185-7.

21. Mackay D, Wood L, Rangan A. The treatment of isolated ulnar fractures in adults: a systematic review. Injury 2000; 31(8): 565-70.

22. Moed BR, Kellam JF, Foster RJ, Tile M, Hansen ST Jr. Immediate internal fixation of open fractures of the diaphysis of the forearm. J Bone Joint Surg Am 1986 Sep; 68(7): 1008-17.

23. Wright RR, Schmeling GI, Schwab JP. The necessity of acute bone grafting in diaphyseal forearm fractures: a retrospective review. J Orthop Trauma 1997 May; 11(4): 288-94.

24. Wei SY, Born CT, Abene A, Ong A, Hayda R, DeLong WG Jr. Diaphyseal forearm fractures treated with and without bone graft. J Trauma 1999; 6: 1045-8

25. Coulibaly MO, Jones CB, Sietsema DL, Schildhauer TA. Results of 70 consecutive ulnar nightstick fractures. Injury 2015 Jul; 46(7): 1359-66. 